\title{
CALIDAD TOTAL EN LOS SISTEMAS DE ENVASE DE ALIMENTOS
}

Eli Espinoza Atencia ${ }^{\prime}$

Deysi Soto Flores ${ }^{2}$

\author{
RES U MEN
}

Se entiende por Calidad Total en Sistemas de Envase de Alimentos, al conjunto de procedimientos adoptados por todos los niveles de la empresa, tendiendo a la utilización adecuada de los envases en la producción de alimentos que consideren las reales necesidades del consumidor a un costo competitivo. El envase contribuye de una forma efectiva a la satisfacción del consumidor, al ofrecer protección a los alimentos, pero especialmente por las razones no tangibles como son el "design", los colores, las informaciones correctas y la practicicidad al abrir y utilizar su contenido.

\section{A B S T R A C T}

By Total Quality in Packaging Systems of Foodstuffs we understand the totality of processes adopted in all the areas of the business, with the object of an appropriate use of packaging in the production of foodstuffs which respond to the real needs of the consumer, at a competitive price. The packaging contributes in an effective way to the satisfaction of the intangible reasons such as the "design", the colours, the correct information and the practicality of opening and using its contents.

1 Doctor en Ingeniería de Alimentos.

2 Ing. pesquero. 


\section{INTRODUCCIÓN}

La Calidad Total es uno de los temas más discutidos y estudiados por las empresas en los últimos años. $\mathrm{Al}$ final, recuperar rápidamente la competitividad perdida en la década pasada y al mismo tiempo atender las expectativas de los clientes, prevenir que ocurran errores o desvíos en relación a las especificaciones de sus productos, comprometer a todos los trabajadores de todos los niveles, promover un gran y continuo programa de entrenamiento y aún más, mantener viva la idea de hacer todo correctamente en la primera vez: ¡No es tarea fácil!

La situación peruana requiere de atención cuando analizamos los datos de paises latinoamericanos, como es el caso de Brasil y los comparamos con las medias mundiales. Así por ejemplo, Brasil invierte en CAPACITACIÓN (entrenamiento) 1\% de sus horas trabajadas (media mundial es de $6 \%$ ), rechaza $2,5 \%$ de los productos que fabrica (media mundial es de $0,02 \%)$, demora 35 días para entregar los pedidos (media mundial es de 3 días) y gasta en asistencia técnica el $2,7 \%$ del valor de las ventas (media mundial es de $0,15 \%)$.

Por los datos presentados anteriormente, se deduce que en el Perú, el problema es aún más grave, por lo que hay un arduo trabajo a ser realizado hasta que los niveles internacionales sean alcanzados y los programas de Calidad Total sean utilizados como herramientas en este sentido. Exigen obviamente inversiones, dedicación y paciencia para reeducar a las personas y adaptarlas a la nueva realidad. Los resultados serán altamente compensadores.

La situación de la industria de alimentos en el Perú no es muy diferente, se caracteriza por tener una amplia diversidad de productos, diferentes grados de tecnologia, variedad en capacidad productiva y perfil de los dirigentes. Entre tanto, todas ellas están conscientes de la vital necesidad de mejores índices productivos que garanticen la propia supervivencia en un mercado cada vez más competitivo.

Otro punto común a todas ellas es el envase, que la gran mayoría entiende apenas como una etapa más de su flujograma de proceso y no como un sistema, y que muchas veces es tan complejo como la propia producción. Los envases mueven millones de dólares en el Perú (Brasil mueve anualmente 6 mil millones de dólares). Es un mercado que se caracteriza por la constante evolución tecnológica con frecuente desarrollo de nuevos materiales. Los materiales de envase inciden entre 3 a $20 \%$ en el precio de venta de los productos alimenticios (la media internacional es de $10 \%)$.
Todos estos datos comprueban la importancia económica del envase y exigen una atención creciente en el estudio de todos los aspectos en ella envueltos (materiales, accesorios, rendimientos, pérdidas, equipamientos, entre otros) con la intención de proponer soluciones amplias para los principales problemas y alternativas de menor costo que contemple el sistema de envase.

En este contexto, el presente trabajo tiene como finalidad presentar una visión sistémica del envase, proporcionar ejemplos de su "modus operandi" típico y discutir las principales prácticas recomendadas para mejorar su calidad total.

\section{CONCEPTOS BÁSICOS}

Se entiende por Calidad Total en Sistemas de Envase de Alimentos, al conjunto de procedimientos adoptados por todos los niveles de la empresa, tendiendo a una adecuada utilización de los envases en la producción de alimentos que atiendan las reales necesidades del consumidor, a un costo competitivo. El envase contribuye de una forma efectiva en la satisfacción del consumidor, al ofrecer protección a los alimentos, pero especialmente por las razones no tangibles como son el "design", los colores, las informaciones correctas y la practicicidad al abrir y utilizar su contenido.

Otras definiciones de calidad también aplicadas al envase son:

\subsection{GARANTÍA DE CALIDAD DE LOS ENVASES}

Es el conjunto de procedimientos adoptados por todos los sectores de la empresa, para establecer, mejorar y asegurar la calidad de la producción en niveles económicos, y satisfacer los deseos de los consumidores.

La garantía de calidad de los envases comienza en la fase de proyecto del producto, asume la forma de control de calidad en la recepción de los envases y en la utilización de los mismos en la producción (pérdidas y rendimientos) y se extiende hasta el producto acabado, evaluando y minimizando el "potencial de los daños" al consumidor.

\subsection{CONTROL DE CALIDAD DE LOS ENVASES}

Es el examen de las características de los envases (atributos y variables) y la verificación del cumplimiento de las especificaciones preestablecidas.

\subsection{CONTROL DEL PROCESO DE ENVASADO}

Es el control de los índices de rendimiento de los 
Página 25

Figura $\mathrm{N}^{0} 1$ :

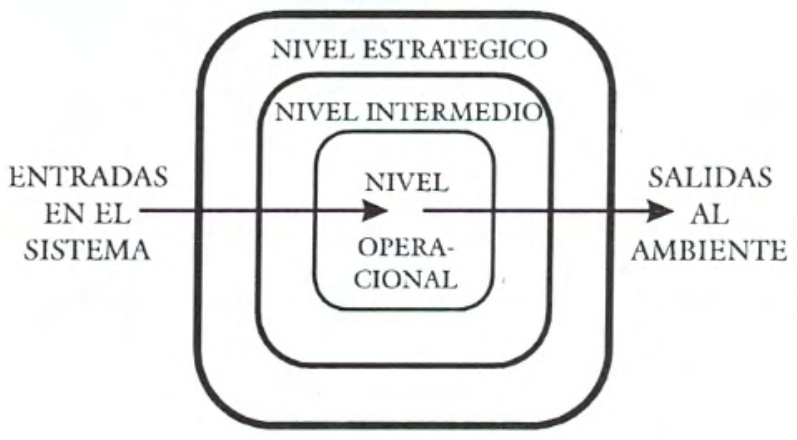

Página 29

Figura $N^{0} 3$

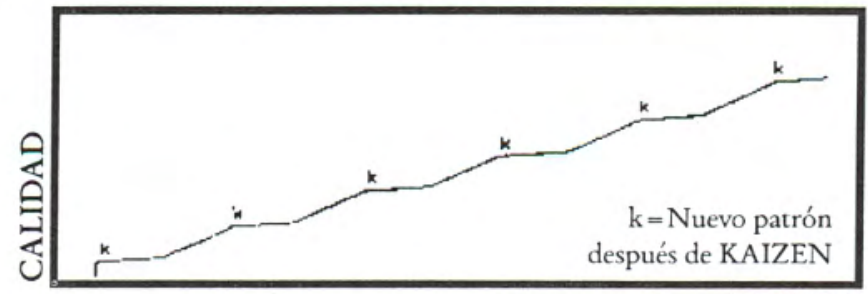

TIEMPO

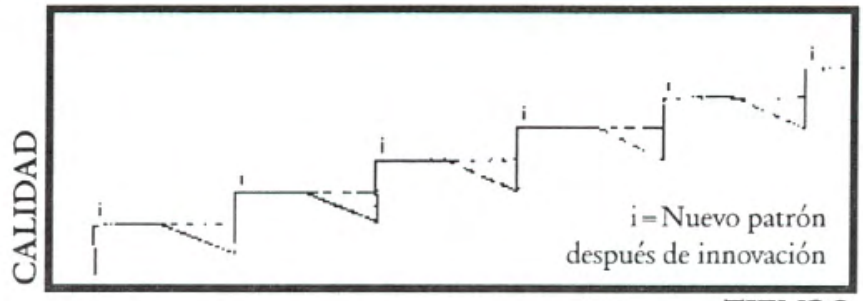

TIEMPO

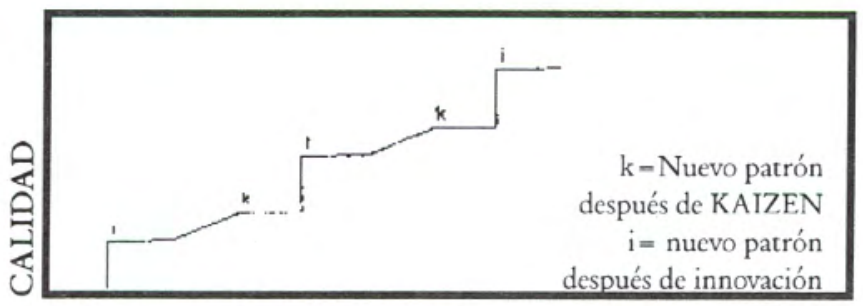

TIEMPO 
equipamientos de envasado y de las pérdidas de materiales, con el objetivo de mantenerlos dentro de los límites de uniformidad establecida en la especificación de fabricación.

\section{4. ÍNDICES DE PRODUCTIVIDAD EN ENVASES}

Está relacionado con el esfuerzo para producir o utilizar envases. Por ejemplo, la productividad de la línea $X$ fue 98 unidades perfectas por cada 100 producidas (98\%).

\section{5. ÍNDICES DE CALIDAD DE ENVASES}

Relacionados a la satisfacción del cliente, como por ejemplo, el porcentaje de lotes rechazados en un mes o el porcentaje de devoluciones de producto en los puntos de venta por envases defectuosos.

\subsection{NIVELES DE LOS SISTEMAS ABIERTOS}

El entendimiento de las definiciones más importantes de calidad es facilitado cuando se percibe a las empresas como sistemas abiertos, absolutamente afectados por la incertidumbre del ambiente que las rodea y sujetos a criterios de racionalidad que garanticen su supervivencia. Estos sistemas abiertos están constituidos de tres principales niveles: institucional (estratégico), intermedio y operacional (Figura №1).

\subsubsection{Nivel institucional o estratégico}

Observa el ambiente externo, trabaja con la incertidumbre a partir de las informaciones obtenidas, toma las decisiones estratégicas de las empresas.

\section{AMBIENTE EXTERNO}

Figura Nro. 1: Los tres principales niveles de los sistemas de envase

\section{ENTRADAS}

EN EL SISTEMA
NIVEL ESTRATÉGICO

NIVEL INTERMEDIO

NIVEL OPERACIONAL

\section{SALIDAS}

$A L$ AMBIENTE

\subsubsection{Nivel intermedio}

Es el nivel medio o gerencial, responsable por la articulación interna institucional/operacional. Transforma las estrategias elaboradas en planes de acción y debe ser por este motivo, flexible y elástico para amortiguar y contener los impactos y presiones externos, sin perjudicar las actividades internas.

\subsubsection{Nivel operacional}

Se da en la racionalidad estrictamente técnica, ejecutando rutinas e ignorando las incertidumbres y variaciones ambientales que son amortiguadas por los otros dos niveles.

Las informaciones expuestas en este item constituyen el fundamento del concepto que se optó por llamar Visión Sistémica del Envase.

\section{VISIÓN SISTÉMICA DEL ENVASE}

Buena parte de las industrias de alimentos consideran al envase sólo como una etapa de su flujograma de proceso o como un simple recipiente para su producto alimenticio y no como un sistema complejo que exige atención permanente. Esto es incorrecto, porque limita la actuación de la empresa apenas al material y deja de lado otros factores que influencian también en su performance.

\subsection{SISTEMA DE ENVASE}

Es el conjunto de operaciones materiales y accesorios que son utilizados en la industria, con la finalidad de contener, proteger y conservar los diversos productos alimenticios y transportarlos a los puntos de venta o utilización, atendiendo las necesidades de los consumidores y/o clientes, a un costo adecuado. Se trata de un sistema abierto, alimentado por necesidades de los consumidores, innovaciones tecnológicas e incertidumbres económicas. Un sistema de envase típico es presentado en la Figura Nro. 2. Analizando cada una de las etapas mencionadas se tiene:

\section{a) Proyecto}

Se sitúa en el nivel estratégico y consiste en la conceptualización del envase. Es desarrollado a partir de la identificación de las necesidades del consumidor o del cliente o a las oportunidades detectadas por el departamento de Márketing. Requiere el conocimiento de las características del producto alimenticio, de su procesamiento, de sus necesidades de protección, de la cadena de comercialización y de los materiales y 
equipamientos disponibles en el mercado.

En la fase de proyecto son realizados todos los ensayos o pruebas que simulen el comportamiento del envase, como permeabilidad, resistencia al transporte y apilamiento, interacciones alimento/materiales de envase y otros.

\section{b) Creación}

También está dentro del nivel estratégico, la creación es simultánea al proyecto y podría igualmente ser considerada parte de ella, si no fuese habitualmente desarrollada por agencias especializadas. Es un proceso que debe ser seguido por el cuerpo técnico de las empresas. Se mezclan la tecnología específica de creación, los "software" avanzados, los estudios sobre el efecto de los colores y las diversas investigaciones, con la capacidad de los profesionales en reunir muchos detalles en una única pieza.

La especificación de los envases, la selección de los proveedores y la compra propiamente dicha de los materiales se localizan en el nivel intermedio.

\section{c) Especificación}

Concluido el proyecto y la creación, se formalizan las propiedades del envase y sus variaciones toleradas en dos documentos que sirven de base para la compra: las especificaciones del material y su plan de control de calidad en la recepción.

\section{d) Selección de proveedores}

Una vez especificado el envase, los departamentos de Compras y Desarrollo seleccionan por criterios técnicos y comerciales (calidad, precio, puntualidad y asistencia técnica o "service"), la empresa que debe abastecer los envases. La preferencia debe ser para aquéllas que ya trabajan con calidad asegurada en el abastecimiento. Muchas veces esta selección es hecha en el inicio del proyecto, asumiendo el carácter de socio.

\section{e) Compra}

La compra es el paso concreto en la materialización del proyecto de envase y debe ser realizada por profesionales capacitados. Todas las empresas deben tener una política de compras bien definida y que considere los criterios ya citados (calidad, precio, puntualidad y asistencia técnica) como indicadores de la salud en la relación proveedor/cliente. Es muy común y debe ser evitada, la compra basada solamente en el precio. Es un enfoque miope porque centra el foco apenas en el precio del material, amplia innecesariamente el abanico de proveedores y no considera a los demás componentes del sistema de envase.

A partir de este punto, se entra al nivel operacional ya definido anteriormente.

\section{f) Recepción}

Los envases adquiridos son recibidos en la fábrica e inspeccionados, siguiendo el plan de control de calidad previamente establecido. En el caso de abastecimiento con calidad asegurada, la inspección es sustituida por un laudo emitido por el fabricante acreditado.

\section{g) Almacenamiento de envases}

Los envases aprobados deben ser almacenados correctamente, obedeciendo al tradicional FIFO (First in-First out) y las recomendaciones técnicas de apilamiento y cuidados en el manejo.

\section{h) Envasado}

A partir de este punto, el envase pasa a confundirse con el producto alimenticio, asumiendo la responsabilidad de contenerlo y protegerlo hasta su consumo o utilización. El concepto de sistema de envase exige un amplio conocimiento de las caracteristicas técnicas y limitaciones de los equipamientos y aplicación práctica de este conocimiento para obtener mejor eficiencia de la línea de producción.

Controlar la performance de los proveedores en el envasado es fundamental para que los rendimientos y pérdidas tengan mejoras continuas. Las distorsiones deben ser evaluadas constantemente por los departamentos de Producción, Control de Calidad y Tecnología de Envases, tratando de identificar sus verdaderas causas, antes que el problema sea transferido al fabricante.

\section{i) Almacenamiento del producto acabado}

Es la última etapa del nivel operacional, el almacenamiento debe respetar las limitaciones del producto (temperatura y humedad relativa adecuados) y del envase (apilamiento y contenerización adecuados).

\section{j) Distribución}

Punto de tránsito para el ambiente externo. La etapa de distribución se sitúa en los niveles estratégicos, como parte de la logística, e intermedio como distribución 
propiamente dicha. Engloba el transporte a los puntos de venta o utilización y el almacenamiento en estos locales hasta el momento de la compra o consumo.

Las operaciones de almacenamiento del producto acabado, distribución y evaluación en los puntos de venta, pueden ser agrupadas en el micro sistema denominado "post-envasado", que debe ser atentamente seguido por la industria productora y por el proveedor de envases, con el objetivo de un continuo perfeccionamiento y la detección de eventuales fallas a corregir. La responsabilidad, en último caso, le cabe a la industria de alimentos.

\subsection{REDUCCIÓN DE COSTOS DEL ENVASE}

En este punto cabe un comentario sobre la reducción de los costos del envase para un determinado producto, bajo la visión sistémica. Los pasos del proceso son los siguientes:

* Cuantificar los costos de cada componente del sistema (Cuadro Nro. 1).

* Identificar los puntos de potencial reducción, usando técnicas específicas.

* Hacer una lista de materiales y accesorios alternativos, los costos de los mismos y las implicancias de su empleo en el sistema. Por ejemplo, reducir el espesor de un envase flexible puede implicar un aumento de la resistencia de la unidad de embarque (caja o saco de papel kraft) para mantener la performance del sistema.

* Corregir los procedimientos inadecuados identificados.

* Evitar las "economías de primer momento" que puedan traer como consecuencia un aumento del costo del sistema. Por ejemplo, transferir la responsabilidad de confección de las artes finales para los proveedores es un gran riesgo porque limita la flexibilidad del márketing para alteraciones y adaptaciones, obliga al departamento de Compras a controlar todas las alteraciones en todos los proveedores y expone a la empresa a un riesgo innecesario de recibir y utilizar envases con textos incorrectos. Solamente el costo de controlar las alteraciones en todos los proveedores ya es mayor que el de la confección del propio arte.

* Volver a calcular el costo del sistema después de las correcciones.

\section{* Volver al primer paso.}

Para reducir costos es, por lo tanto, fundamental entender a los envases como sistema, con procedimientos, operaciones, accesorios y personal técnico específico. Por ejemplo, una determinada caja de embarque puede tener su costo unitario reducido en $6 \%$ por la disminución de su resistencia al apilamiento y este número puede representar una economía de 40,000 dólares/año en el item de envase. Pero el aumento de las pérdidas durante el transporte y almacenamiento en los puntos de venta, por daños mecánicos en el envase primario, prácticamente anula este beneficio y trae como agravante un sensible perjuicio a la imagen del producto. Cabe a la industria, la tarea de controlar permanentemente esto, como respaldo para cualquier alteración en el sistema.

Se toma también como otro ejemplo, el material de envase cuyo costo incida con el $15 \%$ sobre el precio de venta de un producto. Este valor puede llegar a $20 \%$ si consideramos también todas las operaciones y accesorios del sistema (almacenamiento, recepción, análisis, rechazos, perdidas, tarimas o paletas, contenedores, cajas de transporte del material y otros). Es perceptible que el gran beneficio económico puede estar en la mejora de la calidad del sistema y no en la reducción del costo del material en sí.

\section{IV.MEJORAS EN LOS PATRONES DE CALIDAD DEL SISTEMA DE ENVASE}

La palabra "mejora" significa subir uno o más niveles en la evaluación global de la calidad de los sistemas de envase. Se puede dar, tanto en la calidad del material, servicio o proceso utilizado (eficacia), como en su productividad (eficiencia).

El concepto de sistema abierto exige un continuo contacto con el ambiente externo para que todas las informaciones y necesidades sean inmediatamente informadas al ambiente interno, con el objetivo de obtener respuestas bastante rápidas. Exige aún el constante control de todas las etapas, tratando de identificar las causas de los problemas y minimizar sus efectos. Dos tipos de procedimiento pueden ser adoptados :

\section{- Ocasionales}

Tienen objetivos específicos para problemas y/o situaciones muy bien definidas y deben ser interpretados como una región de la estructura a una amenaza. Como ejemplo típico se mencionan las "fuerza tarea".

\section{- Patronizados}

Son aquéllos incorporados o por incorporar en el trabajo cotidiano de las empresas. Engloban técnicas de calidad y de administración industrial y prácticas desarrolladas y mejoradas por las propias empresas.

La única manera de mejorar continuamente el 
sistema, sin dejar de estimular a los trabajadores y manteniendo siempre las puertas abiertas para las innovaciones tecnológicas, es adoptar procedimientos patronizados. Se crea de esta forma, una estructura ágil e inteligente para observar el ambiente externo, trabajar mejor la incertidumbre y responder con vigor a las amenazas.

\subsection{PROCEDIMIENTOS A SER DISCUTIDOS}

\subsubsection{Búsqueda de innovaciones y voluntad de} introducirlas

Las empresas necesitan salir de sus capullos y buscar las innovaciones para sus sistemas de envase. En un momento en que existen programadas por año cerca de 15 exposiciones internacionales de envase, siendo por lo menos 2 de ellas indispensables (la más importante es el Interpack de Alemania), es fácilmente perceptible que los avances en tecnología de envase están ocurriendo a gran velocidad y cualquier vacilación puede ser fatal en el ambiente externo actual, extremadamente competitivo.

La apertura a las importaciones ha facilitado esta búsqueda por innovaciones. El empresario podrá tener contacto directo con equipamientos y materiales innovadores, desplazándose en pocas horas de viaje por numerosos eventos internacionales o por el

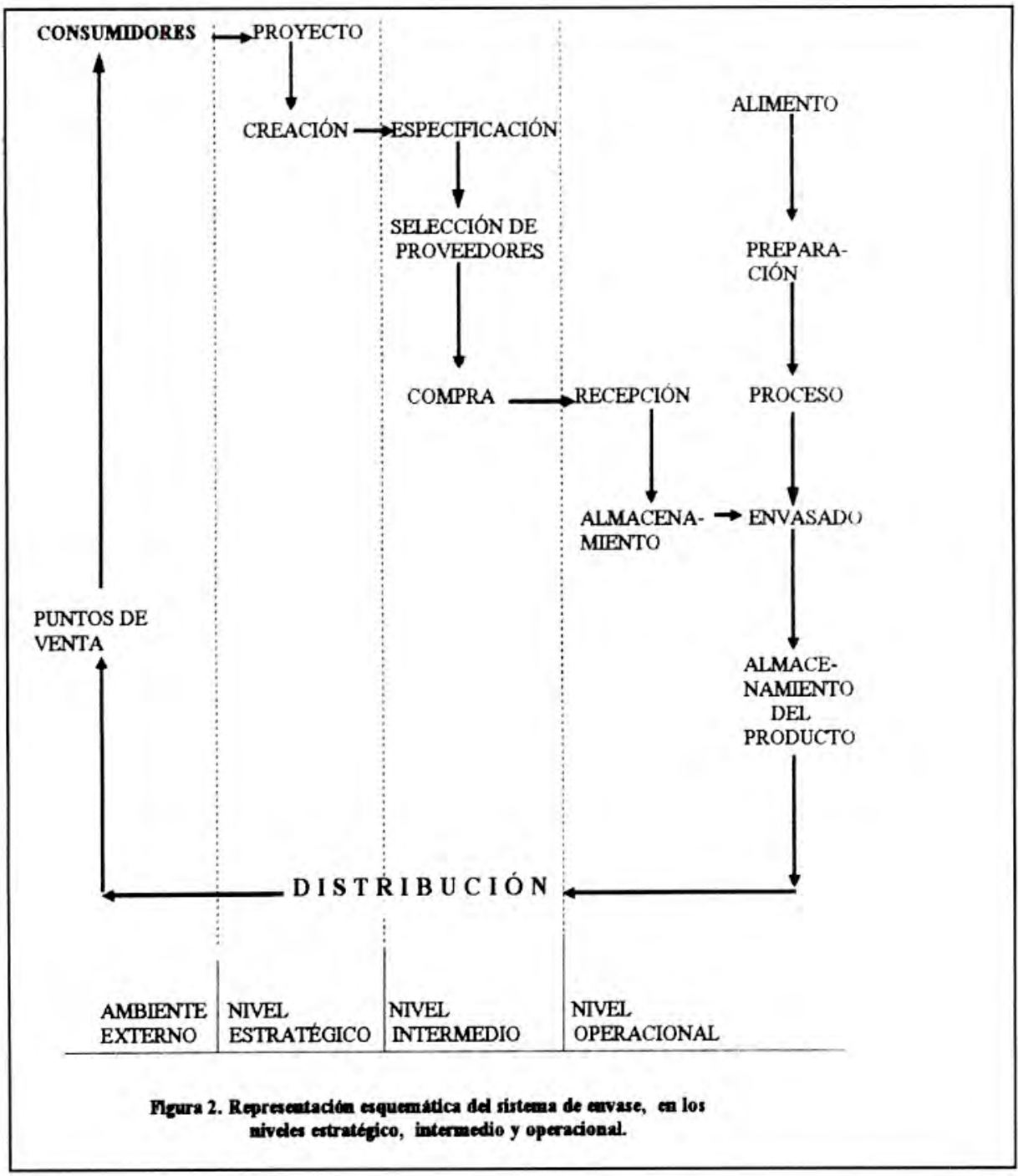

Figura № 2: Representación esquemática del sistema de envase, en los niveles estratégico, intermedio y operacional. 
contacto con una creciente cantidad de empresas que identificarán oportunidades y se podrían instalar en el país (fabricas o representantes técnico-comerciales).

Las innovaciones deben ser introducidas por iniciativa de la propia industria, independiente de su tamaño. Por ejemplo, en Inglaterra el $32,1 \%$ de las innovaciones surgieron en empresas con hasta 200 trabajadores y $33 \%$ de las innovaciones surgieron de proyectos que costaron menos de $\$ 25,000$.

\subsubsection{Mejoras constantes}

El "sistema de envase" abierto a las innovaciones, es caracterizado por las iniciativas de buscarlas, y que al mismo tiempo, persiga siempre los mejores índices de productividad y calidad, para ser finalmente consagrado por el suceso. Su contribución en el resultado de la empresa será por cierto efectiva.

Las mejoras constantes o filosofía KAIZEN, se adaptan muy bien al concepto de sistema de envase: "ningún día debe pasar sin que algún tipo de mejora haya sido hecho en algún lugar de la empresa".

La estrategia KAIZEN reconoce que la administración debe buscar satisfacer al consumidor y atender sus necesidades, si quiere permanecer en los negocios y tener ganancias. Las mejoras constantes en todas las etapas del proceso, por tanto, deben aumentar la satisfacción del consumidor.

En el "sistema de envase", el KAIZEN (que reconoce los esfuerzos de las personas comprometidas en el proceso) significa analizar constantemente cada una de las etapas citadas en la Figura № 2, utilizar los correctos indicadores de calidad y productividad y mejorar los índices continuamente. La Figura № 3 esquematiza situaciones típicas de mejora en empresas que adoptan KAIZEN (3a), innovación (3b) y KAIZEN más innovación (3c), que es considerada la mejor alternativa, porque incorpora los avances tecnológicos y el progreso gradual.

\subsubsection{Benchmarking}

En cuanto el KAIZEN orienta el trabajo en la mejora constante, diaria y algunas veces casi imperceptible, el BENCHMARKING establece objetivos claros de corto, medio y largo plazo para las operaciones del sistema. Por ejemplo, una determinada empresa trabaja con $1,5 \%$ de pérdidas de envases, debe tener como objetivo KAIZEN el de concentrar todos sus esfuerzos para reducir diariamente este índice. EI BENCHMARKING puede establecer el nivel de 0,8\% (el mejor de América Latina) como objetivo a medio plazo y $0,4 \%$ (el mejor del mundo) como meta a largo plazo. Lo importante es que las metas sean establecidas para todas las etapas del sistema.

\subsubsection{Tercerización}

El sistema de envase puede ser tercerizado totalmente, en empresas que no tengan recursos o interés estratégico en ejecutar todas las actividades del sistema, o en algunas de sus etapas como parte de un programa global de reducción de costos. Por ejemplo, se puede transferir para agencias de propaganda el trabajo de crear envases o para el proveedor, la tarea de analizarla, emitiendo certificados y responsabilizándose por los resultados y eventuales costos adicionales (calidad asegurada).

Problemas semejantes tienen tratamientos diferentes en las pequeñas y medianas empresas, cuando son comparadas con empresas grandes. En cuanto éstas utilizan su propio personal, en las primeras la situación es más difícil porque los recursos no siempre están disponibles y ellas acaban entonces recurriendo a terceros (proveedores y agencias).

En suma, la tercerización parcial del sistema de envase en todas las empresas, en mayor o menor escala, es una tendencia muy fuerte para los próximos años.

FIGURA №3: Representación esquemática de las mejoras continuas: (a) apenas KAIZEN; (b) apenas innovación y (c) innovación más KAIZEN.

\section{CALIDAD}

$k=$ Nuevo patrón después de Kaizen

TIEMPO
(a) apenas KAIZEN

CALIDAD

$$
\mathrm{i}=\text { nuevo patrón }
$$

después de innovación

(b) apenas INNOVACIÓN 
CALIDAD

\section{$k=$ Nuevo patrón \\ después de Kaizen \\ $\mathrm{i}$ = nuevo patrón \\ después de innovación}

TIEMPO

(c) INNOVACIÓN más KAIZEN

\section{EL SISTEMA DE ENVASE EN LA INDUSTRIA DE ALIMENTOS (EJEMPLOS)}

Los tres casos que se presentan a continuación, son ejemplos de la aplicación práctica de los conceptos "innovación" y "mejoras constantes", discutidos en este trabajo.

\subsection{EJEMPLO 1: INNOVACIÓN - 1}

A partir de la identificación, realizada por "márketing", de la necesidad del cliente de substituir los envases metálicos de 18 litros para grasas industriales, que presentaban dificultad en el manejo, el "Sistema de Envase" desarrolló e introdujo un nuevo concepto. Las principales actividades ejecutadas en cada una de las etapas fueron:

\section{a) Nivel estratégico}

\section{- Proyecto}

Se identificó la necesidad del cliente, se seleccionó y presentó alternativas de envases adecuado a la vida útil del producto, a las condiciones de manejo y transporte y a la utilización final. La opción recayó sobre bolsas flexibles con $3 \mathrm{~kg}$ de producto, acondicionadas en cajas de embarque.

La introducción de estos envases requirió también la evaluación de equipamientos de envase, pruebas de llenado y de utilización hasta la aprobación final.

\section{- Creación}

Una agencia de propaganda fue encomendada de preparar las artes finales de las cajas de embarque que mantuviesen el patrón actual de las latas e indicasen con claridad las instrucciones de uso. El trabajo fue seguido por los departamentos de Márketing y Tecnología de Envase.

\section{b) Nivel intermedio}

Las actividades de este nivel en la especificación, en la selección de proveedores y compra, fueron muy facilitadas, porque hubo la participación del fabricante de envases y equipamiento desde el inicio del proyecto.

\section{c) Nivel operacional}

\section{- Recepción}

Los envases son abastecidos con calidad asegurada, dispensándose por lo tanto de los análisis de todos los lotes.

\section{- Almacenamiento de los envases}

El material es recibido en forma de bobinas y las cajas de embarque desmontadas, ocupando menor área de almacenamiento, contribuyendo de esta manera en la reducción de los costos del sistema.

\section{- Envasado}

Los índices iniciales de rendimientos y pérdidas se mostraron buenos y se deben tornar aun mejores, en la medida que los operadores ganen más experiencia.

\section{- Almacenamiento del producto acabado}

El aprovechamiento del área de las tarimas o paletas es excepcional (99\%).

La distribución (paso para el ambiente externo) está ocurriendo sin cualquier tipo de problema.

El sistema será continuamente controlado, de modo que se pueda mantener una buena performance inicial.

\subsection{EJEMPLO 2: INNOVACIÓN - 2}

Desarrollo de un nuevo formato de envases para margarina, cuyos principales pasos son:

\section{a) Nivel estratégico}

\section{- Proyecto}

El márketing constató la oportunidad de aumentar los volúmenes de venta del producto, al lanzar una nueva formulación, utilizando un envase innovador.

Las investigaciones con consumidores indicaron que 
dentro de las numerosas alternativas de formatos identificados, la preferencia por el envase ovalado con extremidades truncadas.

\section{- Creación}

Fue desarrollado un trabajo conjuntamente con el departamento de Márketing, Tecnología de Envase, agencia de propaganda y proveedor. Numerosas pruebas de impresión fueron conducidas en los equipamientos del fabricante del envase, hasta lograr la solución que contemple la preferencia del consumidor.

\section{b) Nivel intermedio}

Este trabajo, a ejemplo del citado en 5.1, fue desarrollado conjuntamente con el proveedor de envases, facilitando la ejecución de las etapas de la especificación, selección de proveedores y compras.

\section{c) Nivel operacional}

\section{- Recepción}

Hubo aiteración, apenas en el formato de los envases. Por lo tanto, la metodología de análisis permaneció inalterada.

\section{- Almacenamiento de los envases}

Mantuvo el mismo patrón de los envases redondos tradicionales.

\section{- Envasado}

Exigió adaptaciones en la envasadora y en los procedimientos operacionales, tratando de adecuar esta etapa del sistema al nuevo producto.

\section{- Almacenamiento del producto acabado}

Se mantuvo las exigencias de almacenamiento de las margarinas (temperatura máxima de $16^{\circ} \mathrm{C}$ ), pero exigiendo cuidados adicionales del sistema, por tratarse de un lanzamiento de producto.

En este caso, la distribución requirió un control especifico, hasta que la industria productora pueda obtener informaciones precisas de la performance del producto en los canales de comercialización y efectuar las correcciones necesarias.
Las ventas triplicaron con el nuevo envase. El nivel estratégico controló la performance del producto en los puntos de venta, en el canal de distribución y junto al consumidor para efectuar las correcciones y adaptaciones, eventualmente necesarias.

\subsection{EJEMPLO 3: MEJORAS CONSTANTES}

El caso que claramente ilustra las mejoras constantes es la substitución de películas en equipamientos del tipo "flow pack", con considerable reducción del costo en el sistema. Los pasos seguidos en esas alteraciones fueron:

\section{a) Nivel estratégico}

\section{- Proyecto}

Fue detectada por el departamento de Tecnología de Envase, la posibilidad de substituir películas de policioruro de vinilo (PVC) por polietileno (PE), ambas termoencogibles, sin ningún perjuicio de la imagen del producto ni la satisfacción del consumidor. El cambio trajo sensible reducción en el costo del material del envase primario (40\%) y un ligero incremento en el costo del envase secundario (1\%).

A pesar de la necesidad de adaptaciones en equipamientos, lo cual significó el aporte de recursos, la relación costo/beneficio fue altamente favorable a la introducción de la nueva película.

\section{- Creación}

Nada fue alterado en las artes finales ni en el formato de los envases, porque apenas el material fue cambiado.

\section{b) Nivel intermedio}

\section{- Especificaciones}

Fueron sustituidas las especificaciones de los envases primarios (película) y secundarios (caja de embarque).

\section{- Selección de proveedores}

Fueron evaluados los materiales de tres proveedores potenciales, siendo escogido aquél cuyo material presentó mejor comportamiento en la máquina (velocidad de máquina y pérdidas). 


\section{- Compra}

La compra de envases no sufrió alteraciones en su rutina.

\section{c) Nivel operacional}

\section{- Recepción}

El laboratorio de control de calidad adecuó la metodología de análisis para el nuevo material.

\section{- Almacenamiento de materiales}

El sistema de envase fue favorecido por el cambio del material de envase, porque el polietileno no presenta restricciones de almacenamiento bajo condiciones ambientales, al contrario del PVC termoencogible que no puede ser expuesto a temperaturas mayores de $28^{\circ} \mathrm{C}$.

\section{- Envasado}

Con el nuevo material, la velocidad del envasado y los índices de pérdidas de envases se mantuvieron prácticamente inalterados. Como desventaja, hubo aumento en el consumo de energía en el túnel de encogimiento, sin perjuicio para el balance positivo del sistema.

\section{- Almacenamiento del producto acabado y distribución}

No fue constatada ninguna alteración en estas etapas del sistema.

Después de la substitución del envase se constató una nueva posibilidad de mejora que consistió en la optimización de resinas de polietileno. Los beneficios conseguidos fueron la reducción del costo $(16 \%)$ y de las pérdidas (de $0,6 \%$ para $0,2 \%$ ).

El proceso de mejora continua, tendrá secuencia con ensayos de una nueva composición polimérica, con mayor resistencia mecánica, hecho que al principio obtendrá una mayor velocidad de la máquina y una pequeña ganancia financiera en el material.

\section{CONSIDERACIONES FINALES}

Después de presentar una visión sistémica del envase, dar ejemplos del "modus operandi" típico del sistema y discutir las principales prácticas recomendadas para mejorar su calidad total, un ejercicio final es oportuno.

Una situación ideal futura puede ser imaginada, en la cual las industrias de alimentos hayan incorporado el concepto "sistemático del envase", que comprenda las técnicas de mejora de la calidad, establecida como procedimiento patrón en la búsqueda por innovaciones, obteniendo índices de calidad y productividad semejantes a los de los países desarrollados y tercerizando todas las etapas posibles del sistema. Una pregunta, entonces, podría formularse: ¿qué hacer para diferenciar el "Sistema de Envase" de estas empresas, por hipótesis remodeladas y utilizar consistentemente esta ventaja competitiva?.

\section{Cuadro № 1: Principales componentes de las etapas del sistema de envase.}

\begin{tabular}{|c|c|}
\hline ETAPAS & COMPONENTES \\
\hline Proyecto & $\begin{array}{l}\text { Investigación de mercado } \\
\text { Características del producto } \\
\text { Características de los materiales } \\
\text { Disponibilidad de materiales y } \\
\text { equipamientos } \\
\text { Conocimiento de la cadena de } \\
\text { comercialización } \\
\text { Personal técnico }\end{array}$ \\
\hline Creación & $\begin{array}{l}\text { Agencias (tercerización) } \\
\text { Artes finales } \\
\text { Investigación de mercado/ } \\
\text { aceptación de concepto }\end{array}$ \\
\hline Especificación & $\begin{array}{l}\text { Unidades de acondicionamiento de } \\
\text { envase } \\
\text { Características importantes } \\
\text { Planes de control de calidad } \\
\text { Documentos } \\
\text { Personal técnico }\end{array}$ \\
\hline $\begin{array}{l}\text { Selección de } \\
\text { proveedores }\end{array}$ & $\begin{array}{l}\text { Visitas técnicas/evaluación } \\
\text { Personal técnico }\end{array}$ \\
\hline Compralentrega & $\begin{array}{l}\text { Calidad asegurada } \\
\text { Flete } \\
\text { Uso adecuado de los documentos } \\
\text { y artes finales } \\
\text { Personal técnico }\end{array}$ \\
\hline
\end{tabular}




\begin{tabular}{|c|c|}
\hline Recepción & $\begin{array}{l}\text { Controles de recepción/calidad } \\
\text { asegurada } \\
\text { Equipamientos de laboratorio } \\
\text { Unidades de acondicionamiento de } \\
\text { envase } \\
\text { Personal técnico }\end{array}$ \\
\hline $\begin{array}{l}\text { Almacenamiento } \\
\text { de materiales }\end{array}$ & $\begin{array}{l}\text { Costo/metro cuadrado } \\
\text { Tarimas/porta tarimas } \\
\text { Accesorios retornables } \\
\text { (documentos) } \\
\text { Accesorios no retornables } \\
\text { (descarte) } \\
\text { Pérdidas y daños } \\
\text { FIFO } \\
\text { Personal }\end{array}$ \\
\hline Envasado & $\begin{array}{l}\text { Rendimiento } \\
\text { Pérdidas } \\
\text { Envase secundario (descarte o } \\
\text { reutilización) } \\
\text { Tarimas } \\
\text { Personal técnico }\end{array}$ \\
\hline $\begin{array}{l}\text { Almacenamiento } \\
\text { de producto } \\
\text { acabado }\end{array}$ & $\begin{array}{l}\text { Condiciones de temperatura y } \\
\text { humedad relativa }(\%) \\
\text { Costo/metro cuadrado } \\
\text { Tarimas/porta tarimas } \\
\text { Pérdidas y daños (movimentación } \\
\text { y apilamiento) } \\
\text { Ocupación de las tarimas } \\
\text { (unidades/metro cuadrado) } \\
\text { Ocupación del almacenaje } \\
\text { (unidades/metro cúbico) } \\
\text { Personal }\end{array}$ \\
\hline Distribución & $\begin{array}{l}\text { Flete } \\
\text { Tarimas o carga obtenida } \\
\text { Pérdidas y daños (manejo, } \\
\text { transporte y apilamiento) } \\
\text { Personal }\end{array}$ \\
\hline
\end{tabular}

\begin{tabular}{|l|l|}
\hline Puntos de venta & $\begin{array}{l}\text { Almacenamiento } \\
\text { Manejo } \\
\text { Exposición en góndola (imagen) } \\
\text { Personal }\end{array}$ \\
\hline Consumidor & $\begin{array}{l}\text { Investigación de mercado } \\
\text { (satisfacción con el producto) } \\
\text { Identificación de necesidades }\end{array}$ \\
\hline
\end{tabular}

Deben ser adoptados tres procedimientos:

- Intensificar las prácticas de KAIZEN, independientemente de la tecnología empleada.

- Innovar siempre, en todas la etapas del sistema.

- Encantar al consumidor, ofreciéndole productos de calidad a precios adecuados, acondicionados en envases atrayentes, funcionales, seguros e innovadores.

En realidad, estas recomendaciones pueden ser aplicadas inmediatamente. Lo que varía son los parámetros de calidad que tienden a elevarse en los próximos años.

En cualquier tiempo, presente o futuro, entender el sistema de envase como un sistema abierto y sujeto a las incertidumbres del ambiente externo y poner en práctica este concepto, contribuirá para tornarlo más fuerte, competitivo e innovador. 